\title{
POSITIVE SOLUTIONS \\ OF ONE-DIMENSIONAL $p$-LAPLACIAN EQUATIONS AND APPLICATIONS TO POPULATION MODELS OF ONE SPECIES
}

\author{
Kunquan Lan - XiaOjing Yang - Guangchong Yang
}

\begin{abstract}
We prove new results on the existence of positive solutions of one-dimensional $p$-Laplacian equations under sublinear conditions involving the first eigenvalues of the corresponding homogeneous Dirichlet boundary value problems. To the best of our knowledge, this is the first paper to use fixed point index theory of compact maps to give criteria involving the first eigenvalue for one-dimensional $p$-Laplacian equations with $p \neq 2$. Our results generalize some previous results where either $p$ is required to be greater than 2 or the nonlinearities satisfy stronger conditions. We shall apply our results to tackle a logistic population model arising in mathematical biology.
\end{abstract}

\section{Introduction}

We study the existence of positive (classical) solutions of one-dimensional $p$-Laplacian equations of the form

$$
\left\{\begin{array}{l}
-\Delta_{p} z(x)=f(x, z(x)) \quad \text { for a.e. } x \in(0,1) \\
z(0)=z(1)=0
\end{array}\right.
$$

2010 Mathematics Subject Classification. Primary: 47H10; Secondary: 34B18, 92D25.

Key words and phrases. One-dimensional $p$-Laplacian equations, positive solutions, sublinear condition, fixed point index, logistic population model.

KL was supported in part by the Natural Sciences and Engineering Research Council (NSERC) of Canada, and GY was supported in part by the National Natural Science Foundation of China under grant No. 11171046. 
where $p \in(1, \infty), \Delta_{p} z(x)=\left(\left|z^{\prime}(x)\right|^{p-2} z^{\prime}(x)\right)^{\prime}:=\left(\phi_{p}\left(z^{\prime}(x)\right)\right)^{\prime}, z^{\prime}(x)$ denotes the usual derivative of the function $z$ at $x$, and $\phi_{p}: \mathbb{R} \rightarrow \mathbb{R}$ is defined by

$$
\phi_{p}(s)=|s|^{p-2} s .
$$

One or higher-dimensional $p$-Laplacian equations arise in the study of Newtonian fluids $(p=2)$ and non-Newtonian fluids $(p \neq 2)$ such as dilatant fluids $(p>2)$ and pseudoplastic fluids $(1<p<2)$, for example see Guo and Webb [11] or [27].

Existence of nonzero nonnegative positive solutions of (1.1) has been studied by many authors, for example, by Wang [33], where the nonlinearity is of the form $g(x) f(u)$, under the following condition:

$$
\lim _{u \rightarrow \infty} \frac{f(u)}{u^{p-1}}=0 \quad \text { and } \quad \lim _{u \rightarrow 0+} \frac{f(u)}{u^{p-1}}=\infty,
$$

and by Webb and Lan [36], where $p=2$, under the following sublinear condition:

$$
0 \leq \lim _{u \rightarrow \infty} \frac{f(u)}{u}<\pi^{2}<\lim _{u \rightarrow 0+} \frac{f(u)}{u} \leq \infty .
$$

When $p \geq 2$, Ćwiszewski and Maciejewski [5] use the Granas fixed point index (see [9], [10]) to study the existence of positive weak solutions of $p$-Laplacian equations under the following sublinear condition:

$$
0 \leq \lim _{u \rightarrow \infty} \frac{f(u)}{u^{p-1}}<\mu_{p}<\lim _{u \rightarrow 0+} \frac{f(u)}{u^{p-1}} \leq \infty,
$$

where $\mu_{p}$ is the first eigenvalue of the corresponding homogeneous Dirichlet boundary value problem and $\mu_{2}=\pi^{2}$. Actually [5] also covers the superlinear case and PDE cases, where $f$ is not required to be nonnegative, but [5] only studies weak solutions and requires both a global growth condition on $f$ and $p \geq 2$. Hence, [5] obtained less restrictive solution under stronger assumptions. Rynne [31] studies (1.1) with suitable boundary conditions using bifurcation theory, and Kajikiya, Lee and Sim [15]-[17] study the bifurcation of sign-changing solutions for one-dimensional $p$-Laplacian with a strong singular weight. We refer to [1], [7], [12]-[14], [18], [20]-[25], [29], [32], [37], [38], [41] for the study of the existence and uniqueness of systems of $p$-Laplacian equations under suitable sublinear or superlinear conditions.

In this paper, we obtain new results on the existence of (classical) positive solutions of (1.1) under the sublinear condition (1.3). As mentioned above, there have been many papers studying the existence of solutions of the one-dimensional $p$-Laplacian equation (1.1), but, to the best of our knowledge, when $p \neq 2$, our paper is the first one to use the fixed point index theory of compact maps [2] to give criteria involving the first eigenvalue, which is well known for the case $p=2$, see Webb and Lan [36]. Our results allow $p \in(1, \infty)$ and we obtain positive (classical) solutions in $C_{0}^{1}[0,1]$. Our results generalize Webb and Lan's result in [36] from $p=2$ to $p \neq 2$, Wang's result in [33] from (1.2) to (1.3), 
where the fixed point index theory of compact maps is employed. Our results also improve Ćwiszewski and Maciejewski's result from $p \geq 2$ to $p \in(1, \infty)$ and from weak solutions to classical solutions under the sublinear condition (1.3), where the Granas fixed point index (see [9], [10]) is used to prove the criteria involving the first eigenvalue of $p$-laplacian with $p \geq 2$. We prove our results by applying the theory of fixed point index for compact maps defined on cones in Banach spaces [2]. We overcome the difficulty of lacking linearity of the operator arising from the corresponding homogeneous Dirichlet boundary value problem. Our method is different from those used in [5], where a different index theory is applied, and in [33], [36], where linearity of the corresponding operators are applied in an essential way.

We remark that it remains open whether (1.1) has (classical) positive solutions under the superlinear condition:

$$
0 \leq \lim _{u \rightarrow 0+} \frac{f(u)}{u^{p-1}}<\mu_{p}<\lim _{u \rightarrow \infty} \frac{f(u)}{u^{p-1}} \leq \infty .
$$

As applications of our results, we study the persistence of population models of one species governed by the $p$-Laplacian equations of the form

$$
\left\{\begin{array}{l}
-\Delta_{p} z(x)=\mu z^{\sigma-1}(x)(1-z(x)) \quad \text { for } x \in(0,1), \\
z(0)=z(1)=0
\end{array}\right.
$$

where $z(x)$ denotes the population density of one species at location $x, \mu>0$ is a parameter related to the patch size of the population, the term $z^{\sigma-1}(x)(1-z(x))$ represents the logistic growth rate of order $\sigma$. When $p=\sigma=2,(1.5)$ was studied in [4], [6], [26], [30]. Our result allows $p \in(1, \infty)$ and $\sigma \in(1, p]$.

\section{Positive solutions of one-dimensional $p$-Laplacian equations}

In this section we prove a new result on the existence of nonzero positive solutions of (1.1) and apply it to study the persistence of population models (1.5).

We always assume the following conditions hold:

$\left(\mathrm{C}_{1}\right) f:[0,1] \times \mathbb{R}_{+} \rightarrow \mathbb{R}_{+}$satisfies Carathéodory conditions, that is, $f(\cdot, u)$ is measurable for each fixed $u \in \mathbb{R}_{+}$and $f(t, \cdot)$ is continuous for almost every $t \in[0,1]$.

$\left(\mathrm{C}_{2}\right)$ For each $r>0$ there exists $g_{r} \in L_{+}^{1}(0,1)$ such that

$$
f(x, u) \leq g_{r}(x) \text { for a.e. } x \in[0,1] \text { and all } u \in[0, r] .
$$

The condition $\left(\mathrm{C}_{1}\right)$ is a standard condition which has been widely used, for example in [5], [23]. The upper bound function $g_{r}$ in $\left(\mathrm{C}_{2}\right)$ is independent of $u$ and belongs to $L_{+}^{1}(0,1)$, which is more general than those used previously in [5] and [23]. The condition: $f(x, u) \leq C\left(1+u^{p-1}\right)$ for almost every $x \in[0,1]$ and all $u \in \mathbb{R}_{+}$was used in [5] while [23] required $g_{r}$ in $L_{+}^{\infty}(0,1)$. 
We denote by $A C[0,1]$ the space of all the absolutely continuous functions defined on $[0,1]$.

Definition $2.1([8])$. A function $z:[0,1] \rightarrow \mathbb{R}$ is said to be a (classical) solution of (1.1) if $z \in C^{1}[0,1], \phi_{p}\left(z^{\prime}\right) \in A C[0,1]$ and $z$ satisfies (1.1).

A solution $z$ of (1.1) is said to be nonnegative if $z(x) \geq 0$ for $x \in[0,1]$ and to be positive if $z(x)>0$ for $x \in(0,1)$.

We denote by $W_{0}^{1, p}(0,1)$ the standard Sobolev space with norm

$$
\|u\|_{W_{0}^{1, p}}=\left(\int_{0}^{1}\left|u^{\prime}(x)\right|^{p} d x\right)^{1 / p}:=\left\|u^{\prime}\right\|_{L^{p}},
$$

and by $P$ the positive cone in $W_{0}^{1, p}(0,1)$, that is,

$$
P=\left\{u \in W_{0}^{1, p}(0,1): u(x) \geq 0 \text { for } x \in[0,1]\right\} .
$$

The following result can be found in [19, Lemma A.9(ii), p. 56].

Lemma 2.2. $W_{0}^{1, p}(0,1) \subset C[0,1]$ and there exists $c_{0}>0$ such that

$$
\|u\|_{C[0,1]} \leq c_{0}\|u\|_{W_{0}^{1, p}} \quad \text { for } u \in W_{0}^{1, p} .
$$

We need the following maximum principle, see [28, Lemma 3.1], and the weak comparison principle which is a special case of Lemma 2.4 with $\lambda=0$ in [3].

Lemma 2.3. Assume that a function $u \in C[0,1]$ satisfies the following conditions:

(a) $u^{\prime}(x)$ exists for $x \in(0,1)$ and $\phi_{p}\left(u^{\prime}\right) \in A C(0,1)$.

(b) $-\Delta_{p} u(x) \geq 0$ for a.e. $x \in(0,1)$, and $u(0)=u(1)=0$.

Then $u(x) \geq 0$ for $x \in[0,1]$. If $u \neq \equiv$ on $(0,1)$, then $u(x)>0$ for $x \in(0,1)$.

Lemma 2.4. Assume that $u, w \in W_{0}^{1, p}(0,1)$ satisfy

$$
\left(-\Delta_{p} u(x), v(x)\right) \leq\left(-\Delta_{p} w(x), v(x)\right) \quad \text { for } v \in P,
$$

where

$$
\left(-\Delta_{p} u(x), v(x)\right)=\int_{0}^{1}\left(-\Delta_{p} u(x)\right) v(x) d x .
$$

Then $u(x) \leq w(x)$ almost everywhere on $(0,1)$.

The following result can be found in [39, p. 44].

Lemma 2.5. For every $w \in L^{1}(0,1)$, the quasilinear boundary value problem

$$
\left\{\begin{array}{l}
-\Delta_{p} u(x)=w(x) \quad \text { for a.e. } x \in(0,1), \\
u(0)=u(1)=0,
\end{array}\right.
$$


has a unique solution $u$ in $D\left(\Delta_{p}\right)$, where $D\left(\Delta_{p}\right)=\left\{u \in C_{0}^{1}[0,1]: \phi_{p}\left(u^{\prime}\right) \in\right.$ $A C[0,1]\}$ and $C_{0}^{1}[0,1]=\left\{u \in C^{1}[0,1]: u(0)=u(1)=0\right\}$ is a Banach space with the norm

$$
\|u\|_{C^{1}[0,1]}=\|u\|_{C[0,1]}+\left\|u^{\prime}\right\|_{C[0,1]} .
$$

We denote by $T$ the inverse of $-\Delta_{p}$. Then $T: L^{1}(0,1) \rightarrow D\left(\Delta_{p}\right)$ is defined by

$$
T w=u,
$$

where $u$ is the unique solution of $(2.2)$ in $D\left(\Delta_{p}\right)$.

The following result is a special case of [39, Lemma 1].

Lemma 2.6. The map $T$ defined in (2.4) has the following properties.

(a) $T: L^{1}(0,1) \rightarrow C_{0}^{1}[0,1]$ is continuous and bounded.

(b) $T(B)$ is relatively compact in $C_{0}^{1}[0,1]$ for each subset $B \subset L^{1}(0,1)$ satisfying the following condition:

(C) There exists $h_{B} \in L^{1}(0,1)$ such that

$$
|z(x)| \leq h_{B}(x) \quad \text { for a.e. } x \in(0,1) \text { and each } z \in B .
$$

It is easy to see that the $p$-Laplacian operator $-\Delta_{p}$ has the following property:

$$
\left(-\Delta_{p}\right)(\lambda u(x))=\lambda^{p-1}\left(-\Delta_{p}\right) u(x) \text { for } u \in D\left(\Delta_{p}\right) \text { and } \lambda \geq 0 .
$$

This, together with Lemma 2.5, implies the following property of the inverse operator $T$.

Lemma 2.7. The map $T$ defined in (2.4) has the following property.

$$
T(\lambda w)=\lambda^{1 /(p-1)} T(w) \quad \text { for } w \in L^{1}(0,1) \text { and } \lambda \geq 0 .
$$

Define a map $A$ from $P$ to $D\left(\Delta_{p}\right)$ by

$$
A z(x)=(T F z)(x),
$$

where $T$ is given in (2.4) and the Nemytskii operator $F: C_{+}[0,1] \rightarrow L_{+}^{1}(0,1)$ is defined by

$$
F z(x)=f(x, z(x))
$$

Let $X$ be a Banach space. Recall that a map $A: \Omega \subset X \rightarrow X$ is said to be compact if it is continuous and $\overline{A(D)}$ is compact for each bounded subset $D \subset \Omega$. It is shown in $\left[8\right.$, p. 3] that the map $T$ maps $L^{q}(0,1)$ into $C^{1}[0,1]$ and is compact for $q>1$. Although Lemma $2.6(i)$ shows that $T: L^{1}(0,1) \rightarrow C^{1}[0,1]$ is continuous and bounded, it is not clear whether $T: L^{1}(0,1) \rightarrow C^{1}[0,1]$ is compact.

The following result shows that under the assumptions $\left(\mathrm{C}_{1}\right)$ and $\left(\mathrm{C}_{2}\right)$, the map $A$ defined in (2.5) maps $P$ into $P$ and is compact.

Theorem 2.8. Under $\left(\mathrm{C}_{1}\right)$ and $\left(\mathrm{C}_{2}\right)$, the following assertions hold. 
(a) The map $A$ defined in (2.5) maps $P$ into $P$ and is compact.

(b) $z \in P$ is a fixed point of $A$ if and only if $z$ is a nonnegative solution of (1.1).

Proof. (a) By Lemma 2.2, the embedding map $i_{1}: W_{0}^{1}(0,1) \rightarrow C[0,1]$ defined by $i_{1}(u)=u$ is continuous and hence, $i_{1}: P \rightarrow C_{+}[0,1]$ is continuous. By $\left(\mathrm{C}_{1}\right)$ and $\left(\mathrm{C}_{2}\right), F$ maps $C_{+}[0,1]$ to $L_{+}^{1}(0,1)$ and is continuous. Hence, $F(z)=F\left(i_{1}(z)\right) \in L_{+}^{1}(0,1)$ for $z \in P$, and $F$ maps $P$ into $L_{+}^{1}(0,1)$ and is continuous. Let $w \in L_{+}^{1}(0,1)$ and $u(x)=T w(x)$ for $x \in[0,1]$. By Lemma 2.5 and (2.4), $u \in D\left(\Delta_{p}\right)$ and $u$ satisfies (2.2). Hence, $-\Delta_{p} u(x)=w(x) \geq 0$ for almost every $x \in(0,1)$ and $u(0)=u(1)=0$. By Lemma 2.3, $u(x) \geq 0$ for $x \in(0,1)$ and $u \in P$. Since

$$
\|u\|_{W_{0}^{1, p}} \leq\left\|u^{\prime}\right\|_{C[0,1]} \leq\|u\|_{C^{1}[0,1]} \text { for } u \in C^{1}[0,1],
$$

the embedding map $i_{2}: C_{0}^{1}[0,1] \rightarrow W_{0}^{1, p}(0,1)$ defined by $i_{2}(u)=u$ is continuous. By Lemma $2.6(i), T: L_{+}^{1}(0,1) \rightarrow P$ is continuous and by $(2.5), A: P \rightarrow P$ is continuous. Let $D \subset P$ be bounded. Then there exists $\rho>0$ such that $\|z\|_{W_{0}^{1}} \leq \rho$ for $z \in D$. By Lemma 2.2, $z(x) \leq c_{0} \rho:=r$ for $x \in[0,1]$ and by $\left(\mathrm{C}_{2}\right)$, there exists $g_{r} \in L_{+}^{1}(0,1)$ such that (2.1) holds. By Lemma 2.6(b) with $B=F(D)$ and $h_{B}=g_{r}, A(D)=T(F(D))=T(B)$ is relatively compact in $C_{0}^{1}[0,1]$. Since $i_{2}: C_{0}^{1}[0,1] \rightarrow W_{0}^{1, p}(0,1)$ is continuous, $A(D)=T(B)=i_{2} T(B)$ is relatively compact in $W_{0}^{1}(0,1)$. Hence, $A: P \rightarrow P$ is compact.

(b) Under the assumptions $\left(\mathrm{C}_{1}\right)$ and $\left(\mathrm{C}_{2}\right)$, the results follows from $(2.4)$.

The following result can be found in [40, Theorem 2.1] or [15, Theorem 2.1].

Lemma 2.9. For each $g \in L_{+}^{1}(0,1)$ with $\int_{0}^{1} g(x) d s>0$, there exist $\mu_{g}>0$ and $\varphi_{g} \in C_{0}^{1}[0,1] \cap(P \backslash\{0\})$ satisfying

$$
\left\{\begin{array}{l}
-\Delta_{p} \varphi_{g}(x)=\mu_{g} g(x) \varphi_{g}^{p-1}(x) \quad \text { for a.e. } x \in(0,1), \\
\varphi_{g}(0)=\varphi_{g}(1)=0 .
\end{array}\right.
$$

The positive value $\mu_{g}$ is called the first eigenvalue of $(2.7)$ and $\varphi_{g}$ is called the eigenfunction corresponding to the eigenvalue $\mu_{g}$. By [40, (3.5), p. 42], we see that for each $g \in L_{+}^{1}(0,1) \backslash\{0\}$,

$$
\mu_{g}=\inf \left\{\int_{0}^{1}\left|u^{\prime}(x)\right|^{p} d x / \int_{0}^{1} g(x)|u(x)|^{p} d x: u \in W_{0}^{1, p}(0,1) \backslash\{0\}\right\} .
$$

where $\int_{0}^{1}\left|u^{\prime}(x)\right|^{p} d x / \int_{0}^{1} g(x)|u(x)|^{p} d x=\infty$ if $\int_{0}^{1} g(x)|u(x)|^{p} d x=0$. It is given in $[8,(3.8)]$ that the first eigenvalue $\mu_{g}$ with $g \equiv 1$ equals

$$
\mu_{1}(p):=\left\{2 \int_{0}^{(p-1)^{1 / p}}\left[1-s^{p}(p-1)^{-1}\right]^{-1 / p} d s\right\}^{p} .
$$


Let $r>0$ and let $P_{r}=\{x \in P:\|x\|<r\}, \partial P_{r}=\{x \in P:\|x\|=r\}$ and $\bar{P}_{r}=\{x \in P:\|x\| \leq r\}$.

Lemma 2.10 ([2]).

(a) If $A: \bar{P}_{r} \rightarrow P$ is compact and satisfies $z \neq t A z$ for $x \in \partial P_{r}$ and $t \in(0,1]$, then $i_{P}\left(A, P_{r}\right)=1$.

(b) If $A: \bar{P}_{r} \rightarrow P$ is compact and $z \neq A z$ for $z \in \bar{P}_{r}$, then $i_{P}\left(A, P_{r}\right)=0$.

(c) Assume that $h:[0,1] \times \bar{P}_{r} \rightarrow P$ is compact and satisfies $z \neq h(t, z)$ for $(t, z) \in[0,1] \times \partial P_{r}$. Then $i_{P}\left(h(0, \cdot), P_{r}\right)=i_{P}\left(h(1, \cdot), P_{r}\right)$.

(d) If $i_{P}\left(A, P_{r}\right)=1$ and $i_{P}\left(A, P_{\rho}\right)=0$ for some $\rho \in(0, r)$, then $A$ has a fixed point in $P_{r} \backslash \bar{P}_{\rho}$.

Now, we state and prove our main result.

Theorem 2.11. Assume that $\left(\mathrm{C}_{1}\right),\left(\mathrm{C}_{2}\right)$ and the following conditions hold:

$\left(\mathrm{H}_{1}\right)$ There exist $r_{0}>0, \varepsilon>0$ and $\phi_{r_{0}} \in L_{+}^{1}(0,1) \backslash\{0\}$ such that

$$
f(x, u) \leq\left(\mu_{\phi_{r_{0}}}-\varepsilon\right) \phi_{r_{0}}(x) u^{p-1} \quad \text { for a.e. } x \in[0,1] \text { and all } u \in\left[r_{0}, \infty\right) .
$$

$\left(\mathrm{H}_{2}\right)$ There exist $\rho_{0}>0, \varepsilon>0$ and $\psi_{\rho_{0}} \in L_{+}^{1}(0,1) \backslash\{0\}$ such that

$$
f(x, u) \geq\left(\mu_{\psi_{\rho_{0}}}+\varepsilon\right) \psi_{\rho_{0}}(x) u^{p-1} \quad \text { for a.e. } x \in[0,1] \text { and all } u \in\left[0, \rho_{0}\right] .
$$

Then (1.1) has a positive solution $z$ in $C_{0}^{1}[0,1]$, that is, $z \in C_{0}^{1}[0,1]$ satisfies $z(x)>0$ for $x \in(0,1)$.

Proof. By $\left(\mathrm{C}_{2}\right)$, there exists $g_{r_{0}} \in L_{+}^{1}(0,1)$ such that

$$
f(x, u) \leq g_{r_{0}}(x) \text { for a.e. } x \in[0,1] \text { and all } u \in\left[0, r_{0}\right] .
$$

This, together with $\left(\mathrm{H}_{1}\right)$, implies, for almost every $x \in[0,1]$ and all $u \in \mathbb{R}_{+}$,

$$
f(x, u) \leq g_{r_{0}}(x)+\left(\mu_{\phi_{r_{0}}}-\varepsilon\right) \phi_{r_{0}}(x) u^{p-1} .
$$

Let $r_{1}=\left(\varepsilon^{-1} c_{0} \mu_{\phi_{r_{0}}}\left\|g_{r_{0}}\right\|_{L^{1}}\right)^{1 /(p-1)}$ and $r>\max \left\{r_{1}, c_{0}^{-1} \rho_{0}\right\}$. We prove that

$$
z \neq t A z \quad \text { for } z \in \partial P_{r} \text { and } t \in[0,1] .
$$

In fact, if not, there exist $z \in \partial P_{r}$ and $t \in(0,1]$ such that $z=t A z$. By $(2.5)$ and Lemma 2.7, $z(x)=T\left(t^{p-1} F z\right)(x)$ for $x \in[0,1]$. It follows from (2.4) that

$$
-\Delta_{p} z(x)=t^{p-1} f(x, z(x)) \text { for a.e. } x \in[0,1] .
$$


By (2.12), (2.10), (2.8) with $g=\phi_{r_{0}}$ and Lemma 2.2, we have

$$
\begin{aligned}
\|z\|_{W_{0}^{1, p}}^{p} & =\left(-\Delta_{p} z, z\right)=t^{p-1} \int_{0}^{1} f(x, z(x)) z(x) d x \leq \int_{0}^{1} f(x, z(x)) z(x) d x \\
& \leq \int_{0}^{1}\left[g_{r_{0}}(x)+\left(\mu_{\phi_{r_{0}}}-\varepsilon\right) \phi_{r_{0}}(x) z^{p-1}(x)\right] z(x) d x \\
& \leq\|z\|_{C[0,1]}\left\|g_{r_{0}}\right\|_{L^{1}}+\left(\mu_{\phi_{r_{0}}}-\varepsilon\right) \mu_{\phi_{r_{0}}}^{-1}\|z\|_{W_{0}^{1, p}}^{p} \\
& \leq c_{0}\|z\|_{W_{0}^{1, p}}\left\|g_{r_{0}}\right\|_{L^{1}}+\left(1-\varepsilon \mu_{\phi_{r_{0}}}^{-1}\right)\|z\|_{W_{0}^{1, p}}^{p} .
\end{aligned}
$$

This implies that $\|z\|_{W_{0}^{1, p}}^{p-1} \leq \varepsilon^{-1} c_{0} \mu_{\phi_{r_{0}}}\left\|g_{r_{0}}\right\|_{L^{1}}$. Hence, we have

$$
r_{1}<r=\|z\|_{W_{0}^{1, p}} \leq\left(\varepsilon^{-1} c_{0} \mu_{\phi_{r_{0}}}\left\|g_{r_{0}}\right\|_{L^{1}}\right)^{1 /(p-1)}=r_{1},
$$

a contradiction. By (2.11) and Lemma 2.10(a), $i_{P}\left(A, P_{r}\right)=1$.

Let $\rho=c_{0}^{-1} \rho_{0}$. Then $\rho<r$. By Lemma 2.2, we have for $z \in \partial P_{\rho}$,

$$
z(x) \leq\|z\|_{C[0,1]} \leq c_{0}\|z\|_{W_{0}^{1, p}}=c_{0} \rho=\rho_{0} \quad \text { for } x \in[0,1] .
$$

It follows from $\left(\mathrm{H}_{2}\right)$ that for almost every $x \in[0,1]$ and all $z \in \partial P_{\rho}$,

$$
f(x, z(x)) \geq\left(\mu_{\psi_{\rho_{0}}}+\varepsilon\right) \psi_{\rho_{0}}(x) z^{p-1}(x) .
$$

If there exists $z \in \partial P_{\rho}$ such that $z=T(F z)$, then the result of Theorem 2.11 holds. Hence, we assume that $z \neq T(F z)$ for $z \in \partial P_{\rho}$ and prove that

$$
z \neq T\left(F z+\nu\left(-\Delta_{p} e\right)\right) \quad \text { for } z \in \partial P_{\rho} \text { and } \nu>0,
$$

where $e$ is the eigenfunction corresponding to the eigenvalue $\mu_{\psi_{\rho_{0}}}$, that is,

$$
\left\{\begin{array}{l}
-\Delta_{p} e(x)=\mu_{\psi_{\rho_{0}}} \psi_{\rho_{0}}(x) e^{p-1}(x) \quad \text { for a.e. } x \in(0,1), \\
e(0)=e(1)=0
\end{array}\right.
$$

In fact, if not, there exist $z \in \partial P_{\rho}$ and $\nu>0$ such that $z=T\left(F z+\nu\left(-\Delta_{p} e\right)\right)$. Then

$$
-\Delta_{p} z(x)=F z(x)+\nu\left(-\Delta_{p} e\right)(x) \text { for a.e. } x \in[0,1]
$$

and we have for $v \in P$,

$$
\left(-\Delta_{p} z, v\right)=(F z, v)+\nu\left(\left(-\Delta_{p} e\right), v\right) \geq \nu\left(\left(-\Delta_{p} e\right), v\right)=\left(-\Delta_{p}\left(\nu^{1 /(p-1)} e\right), v\right) .
$$

By Lemma 2.4 and continuity of $z$ and $e, z(x) \geq \nu^{\frac{1}{p-1}} e(x)$ for $x \in(0,1)$. Let

$$
\tau=\sup \left\{\zeta>0: z(x) \geq \zeta^{1 /(p-1)} e(x) \quad \text { for } x \in(0,1)\right\} .
$$

Then $0<\nu \leq \tau<\infty$ and

$$
z(x) \geq \tau^{1 /(p-1)} e(x) \quad \text { for } x \in(0,1) .
$$


By (2.15), we see that $\left(\left(-\Delta_{p} e\right), v\right) \geq 0$ for $v \in P$. By (2.16), (2.13), (2.18) and (2.15), we have for $v \in P$,

$$
\begin{aligned}
\left(-\Delta_{p} z, v\right) & =\int_{0}^{1} f(x, z(x)) v(x) d x+\nu\left(\left(-\Delta_{p} e\right), v\right) \geq \int_{0}^{1} f(x, z(x)) v(x) d x \\
& \geq\left(\mu_{\psi_{\rho_{0}}}+\varepsilon\right) \int_{0}^{1} \psi_{\rho_{0}}(x) z^{p-1}(x) v(x) d x \\
& \geq\left(\mu_{\psi_{\rho_{0}}}+\varepsilon\right) \tau \int_{0}^{1} \psi_{\rho_{0}}(x) e^{p-1}(x) v(x) d x \\
& =\xi \int_{0}^{1} \mu_{\psi_{\rho_{0}}} \psi_{\rho_{0}}(x) e^{p-1}(x) v(x) d x \\
& =\xi\left(-\Delta_{p} e, v\right)=\left(-\Delta_{p}\left(\xi^{1 /(p-1)} e\right), v\right),
\end{aligned}
$$

where $\xi=\left(\mu_{\psi_{\rho_{0}}}+\varepsilon\right) \tau \mu_{\psi_{\rho_{0}}}^{-1}$. Using Lemma 2.4 and continuity of $z$ and $e$, we obtain

$$
z(x) \geq \xi^{1 /(p-1)} e(x) \quad \text { for } x \in(0,1) .
$$

By (2.17), we have $\tau \geq \xi=\left(\mu_{\psi_{\rho_{0}}}+\varepsilon\right) \tau \mu_{\psi_{\rho_{0}}}^{-1}>\tau$, a contradiction.

Now, we prove that there exists $n_{0} \in \mathbb{N}$ such that

$$
z \neq T\left(F z+n_{0}\left(-\Delta_{p} e\right)\right) \text { for } z \in \bar{P}_{\rho} .
$$

In fact, if not, there exists $z_{n} \in \bar{P}_{\rho}$ such that $z_{n}=T\left(F z_{n}+n\left(-\Delta_{p} e\right)\right)$. It follows from Lemma 2.7 that

$$
\frac{z_{n}}{n^{1 /(p-1)}}=T\left(\frac{F z_{n}}{n}+\left(-\Delta_{p} e\right)\right)
$$

By Lemma 2.2,

$$
z_{n}(x) \leq\left\|z_{n}\right\|_{C[0,1]} \leq c_{0}\left\|z_{n}\right\|_{W_{0}^{1, p}}=c_{0} \rho \text { for } x \in[0,1] .
$$

By $\left(\mathrm{C}_{2}\right)$, there exists $g_{c_{0} \rho} \in L_{+}^{1}(0,1)$ such that

$$
f\left(x, z_{n}(x)\right) \leq g_{c_{0} \rho}(x) \text { for a.e. } x \in[0,1] .
$$

Hence, we have

$$
\left\|\frac{F z_{n}}{n}\right\|_{L^{1}}=\frac{1}{n} \int_{0}^{1} f\left(x, z_{n}(x)\right) d x \leq \frac{1}{n} \int_{0}^{1} g_{c_{0} \rho}(x) d x=\frac{\left\|g_{c_{0} \rho}\right\|_{L^{1}}}{n} \rightarrow 0
$$

and

$$
\frac{F z_{n}}{n}+\left(-\Delta_{p} e\right) \rightarrow-\Delta_{p} e \quad \text { in } L^{1}(0,1) .
$$

By $(2.15),-\Delta_{p} e \in L_{+}^{1}(0,1)$ and $F z_{n} / n+\left(-\Delta_{p} e\right) \in L_{+}^{1}(0,1)$. By Lemma 2.6(a),

$$
T\left(\frac{F z_{n}}{n}+\left(-\Delta_{p} e\right)\right) \rightarrow T\left(-\Delta_{p} e\right) \text { in } C_{0}^{1}[0,1] .
$$

Since the identity $i_{2}: C_{0}^{1}[0,1] \rightarrow W_{0}^{1, p}(0,1)$ defined by $i_{2}(u)=u$ is continuous,

$$
T\left(\frac{F z_{n}}{n}+\left(-\Delta_{p} e\right)\right) \rightarrow T\left(-\Delta_{p} e\right) \text { in } W_{0}^{1, p}(0,1) .
$$


Since $z_{n} \in \bar{P}_{\rho}$, we have $z_{n} / n^{1 /(p-1)} \rightarrow 0$ in $W_{0}^{1, p}(0,1)$. By $(2.20)$ and $(2.21)$, we have $0=T\left(-\Delta_{p} e\right)=e$, which contradicts the fact that the eigenfunction $e$ is nonzero.

Using (2.19) and Lemma 2.10(a), we have

$$
i_{P}\left(T\left(F z+n_{0}\left(-\Delta_{p} e\right)\right), P_{\rho}\right)=0 .
$$

We define a map $h:[0,1] \times \bar{P}_{\rho} \rightarrow P$ by

$$
h(t, z)=T\left(F z+n_{0} t\left(-\Delta_{p} e\right)\right) .
$$

Then $h:[0,1] \times \bar{P}_{\rho} \rightarrow P$ is compact and by $(2.14), z \neq h(t, z)$ for $(t, z) \in$ $[0,1] \times \partial P_{\rho}$. By Lemma $2.10(\mathrm{c})$, we obtain

$$
i_{P}\left(h(0, \cdot), P_{\rho}\right)=i_{P}\left(h(1, \cdot), P_{\rho}\right) .
$$

It follows from (2.23) and (2.22) that

$i_{P}\left(A, P_{\rho}\right)=i_{P}\left(h(0, \cdot), P_{\rho}\right)=i_{P}\left(h(1, \cdot), P_{\rho}\right)=i_{P}\left(T\left(F z+n_{0}\left(-\Delta_{p} e\right)\right), P_{\rho}\right)=0$.

By Lemma $2.10(i v)$, there exists $z \in P_{r} \backslash \bar{P}_{\rho}$ such that $z=A z$ and by Theorem $2.8, z$ is a nonnegative solution of (1.1). By Lemma $2.6(i), T$ maps $L^{1}(0,1)$ into $C_{0}^{1}[0,1]$ and thus, $z \in C_{0}^{1}[0,1] \backslash\{0\}$. It follows from Lemma 2.3 that $z(x)>0$ for $x \in(0,1)$.

REMARK 2.12. Theorem 2.11 allows $f$ to have explicit dependence on $x$. We refer to Webb [34], [35] for work on semi-linear problems, which includes the case $p=2$ here, when $f$ depends explicitly on $x$.

Let $E$ be a fixed subset of $[0,1]$ of measure zero. Let

$$
\begin{array}{ll}
\underline{f}(z)=\inf _{x \in[0,1] \backslash E} f(x, z), & \left(f_{p}\right)_{0}=\liminf _{z \rightarrow 0+} \underline{f}(z) / z^{p-1}, \\
\bar{f}(z)=\sup _{x \in[0,1] \backslash E} f(x, z), & f_{p}^{\infty}=\limsup _{z \rightarrow \infty} \bar{f}(z) / z^{p-1} .
\end{array}
$$

As a special case of Theorem 2.11, we give the following result which depends on the behavior of $f(z) / z^{p-1}$ at 0 and $\infty$.

Corollary 2.13. Assume that $\left(\mathrm{C}_{1}\right),\left(\mathrm{C}_{2}\right)$ and the following condition holds:

$$
0 \leq f_{p}^{\infty}<\mu_{1}(p)<\left(f_{p}\right)_{0} \leq \infty,
$$

where $\mu_{1}(p)$ is the same as in (2.9). Then (1.1) has a positive solution $z$ in $C_{0}^{1}[0,1]$.

Proof. By $(2.24),\left(\mathrm{H}_{1}\right)$ with $\phi_{r_{0}} \equiv 1$ and $\left(\mathrm{H}_{2}\right)$ with $\psi_{\rho_{0}} \equiv 1$ hold for some $\varepsilon>0$ and $\rho_{0}, r_{0}$ with $0<\rho_{0}<r_{0}<\infty$. The result follows from Theorem 2.11. 
Remark 2.14. Corollary 2.13 improves [5, Theorem 1.1] with $N=1$ and $\rho_{\infty}(x)<\lambda_{1, p}<\rho_{\infty}(x)$ in the following ways:

(a) The condition (b) in [5, Theorem 1.1] is stronger than the condition $\left(\mathrm{C}_{2}\right)$ in this paper.

(b) Corollary 2.13 allows $p \in(1, \infty)$ while [5, Theorem 1.1] requires $p \geq 2$.

(c) The nonzero negative solution $z$ in Corollary 2.13 is a classical solution (see Definition 2.1) while the solution $z$ in [5, Theorem 1.1] is a weak solution, that is, $z$ satisfies

$$
\int_{0}^{1} \phi_{p}\left(z^{\prime}(x)\right) z^{\prime}(x) d x=\int_{0}^{1} f(x, z(x)) z(z) d x \quad \text { for } z \in W_{0}^{1}(0,1) .
$$

It is known that if $f(\cdot, z(\cdot))$ is in $L^{r}$, where $r>1$, then the weak solution $z$ is a classical solution. (see $\left[8\right.$, p. 3]). However, under the condition $\left(\mathrm{C}_{2}\right)$, we see that $f(\cdot, z(\cdot))$ is in $L^{1}(0,1)$. Hence, under the condition $\left(\mathrm{C}_{2}\right)$, it is not clear whether a weak solution of (1.1) is a classical solution.

(d) Corollary 2.13 does not require the two limits $\lim _{|z| \rightarrow 0+} f(x, z) / z^{p-1}$ and $\lim _{z \rightarrow \infty} f(x, z) / z^{p-1}$ converge uniformly on $[0,1]$.

(e) Our method is different from that used in [5].

The function $f$ in Corollary 2.13 depends on $x$, but if $f$ is independent of $x$, then we have the following result.

Corollary 2.15. Assume that $f: \mathbb{R}_{+} \rightarrow \mathbb{R}_{+}$is continuous and satisfies

$$
0 \leq \limsup _{u \rightarrow \infty} \frac{f(u)}{u^{p-1}}<\mu_{1}(p)<\liminf _{u \rightarrow 0+} \frac{f(u)}{u^{p-1}} \leq \infty .
$$

Then the following p-Laplacian equation

$$
\left\{\begin{array}{l}
-\Delta_{p} z(x)=f(z(x)) \quad \text { for } x \in(0,1), \\
z(0)=z(1)=0
\end{array}\right.
$$

has a positive solution $z$ in $C_{0}^{1}[0,1]$ satisfying $z(x)>0$ for $x \in(0,1)$.

As another special case of Theorem 2.11, we obtain the following result.

Corollary 2.16. Assume that $g \in L_{+}^{1}(0,1)$ with $\int_{0}^{1} g(s) d s>0$ and $f:[0,1]$ $\times \mathbb{R}_{+} \rightarrow \mathbb{R}_{+}$satisfies $\left(\mathrm{C}_{1}\right),\left(\mathrm{C}_{2}\right)$ with $g_{r} \in L_{+}^{\infty}(0,1)$, and $(2.24)$. Then the following $p$-Laplacian equation

$$
\left\{\begin{array}{l}
-\Delta_{p} z(x)=g(x) f(x, z(x)) \quad \text { for } x \in(0,1), \\
z(0)=z(1)=0 .
\end{array}\right.
$$

has a positive solution $z$ in $C_{0}^{1}[0,1]$ satisfying $z(x)>0$ for $x \in(0,1)$.

Proof. Since $g \in L^{1}(0,1)$ and $g_{r} \in L_{+}^{\infty}(0,1)$, the product $g f$ of $g$ and $f$ satisfies $\left(\mathrm{C}_{2}\right)$. The rest of the proof is similar to that of Corollary 2.13. 
REMARK 2.17. Corollary 2.16 with $p=2$ was essentially obtained by Webb and Lan [36], see Theorem 4.1 $\left(\mathrm{H}_{2}\right)$ and Theorem 5.1(a) in [36]. Corollary 2.16 with $p \neq 2$ improves Theorem 3(b) with the Dirichlet boundary condition in [33], where $f$ is independent of $x, \lim _{u \rightarrow \infty} f(u) / u^{p-1}=0$ and $\lim _{u \rightarrow 0+} f(u) / u^{p-1}=\infty, g$ satisfies a stronger condition, see $\left(1.6_{a}\right)$ in [33].

\section{Applications to persistence of population models of one species}

In this section, we apply the results in Section 2 to study the persistence of population models of one species governed by (1.5), that is,

$$
\left\{\begin{array}{l}
-\Delta_{p} z(x)=\mu z^{\sigma-1}(x)(1-z(x)) \text { for } x \in(0,1), \\
z(0)=z(1)=0
\end{array}\right.
$$

where $z(x)$ denotes the population density of one species at location $x, \mu>0$ is a parameter related to the patch size of the population, the term $z^{\sigma-1}(x)(1-$ $z(x))$ represents the logistic growth rate of order $\sigma$. We refer to [4], [6], [26], [30] for the study of (3.1) with $p=\sigma=2$. Here we allow $p \in(1, \infty)$ and $\sigma \in(1, p]$. To make the population persist on every location $x \in(0,1)$, one needs to find a positive solution $z$.

Let $p \in(1, \infty)$ and let

$$
\mu(\sigma)= \begin{cases}\mu_{p} & \text { if } \sigma=p, \\ 0 & \text { for } \sigma \in(1, p) .\end{cases}
$$

Theorem 3.1. Let $p \in(1, \infty)$ and $\sigma \in(1, p]$. Then for $\mu \in(\mu(\sigma), \infty),(3.1)$ has a positive solution $z$ in $C_{0}^{1}[0,1]$.

Proof. Let $\mu \in(\mu(\sigma), \infty)$. We define a function $\mathbb{R}_{+} \rightarrow \mathbb{R}_{+}$by

$$
f(u)= \begin{cases}\mu u^{\sigma-1}(1-u) & \text { for } u \in[0,1], \\ 0 & \text { for } u \in(1, \infty) .\end{cases}
$$

Then $\lim _{u \rightarrow \infty} f(u) / u^{p-1}=0<\mu_{p}$ and

$$
\lim _{u \rightarrow 0^{+}} \frac{f(u)}{u^{p-1}}=\mu \lim _{u \rightarrow 0^{+}} \frac{1}{u^{p-\sigma}} \lim _{u \rightarrow 0^{+}}(1-u)=\left\{\begin{array}{ll}
\mu & \text { if } \sigma=p, \\
\infty & \text { for } \sigma \in(1, p),
\end{array}>\mu_{p} .\right.
$$

By Corollary $2.15,(2.25)$ with the function $f$ defined in (3.3) has a positive solution $z$ in $C_{0}^{1}[0,1]$ satisfying $z(x)>0$ for $x \in(0,1)$. We prove that $z$ is a solution of (3.1). It suffices to prove that $\|z\|_{C[0,1]} \leq 1$. The proof is by contradiction. In fact, if $\|z\|_{C[0,1]}>1$, then there exists $x_{0} \in(0,1)$ such that 
$z\left(x_{0}\right)=\|z\|_{C[0,1]}>1$. It follows that there exists $\delta \in\left(0, \min \left\{x_{0}, 1-x_{0}\right\}\right)$ such that $z(x)>1$ for $x \in\left(x_{0}-\delta, x_{0}+\delta\right)$. Let

$$
x_{1}=\inf \left\{x \in[0,1]: z(s)>1 \text { for } s \in\left[x, x_{0}\right]\right\}
$$

and

$$
x_{2}=\sup \left\{x \in[0,1]: z(s)>1 \text { for } s \in\left[x_{0}, x\right]\right\} .
$$

Then the following properties hold: (i) $0<x_{1}<x_{0}<x_{2}<1$, (ii) $z(x)>1$ for $x \in\left(x_{1}, x_{2}\right)$, (iii) $z\left(x_{1}\right)=z\left(x_{2}\right)=1$ and (iv) $z^{\prime}\left(x_{0}\right)=0$. By (3.3), $f(z(x))=0$ for $x \in\left[x_{1}, x_{2}\right]$. Hence,

$$
-\Delta_{p} z(x)=f(z(x))=0 \quad \text { for } x \in\left[x_{1}, x_{2}\right] .
$$

This implies that there exists a constant $\eta>0$ such that

$$
\phi_{p}\left(z^{\prime}(x)\right)=\left|z^{\prime}(x)\right|^{p-2} z^{\prime}(x)=\eta \quad \text { for } x \in\left[x_{1}, x_{2}\right] .
$$

Since $\phi_{p}: \mathbb{R} \rightarrow \mathbb{R}$ is strictly increasing on $\mathbb{R}, z^{\prime}(x)=\phi_{p}^{-1}(\eta)=\phi_{q}(\eta)$ for $x \in$ $\left[x_{1}, x_{2}\right]$, where $1 / p+1 / q=1$. Since $z^{\prime}\left(x_{0}\right)=0$ and $x_{0} \in\left[x_{1}, x_{2}\right]$, we have $z^{\prime}(x)=0$ for $x \in\left[x_{1}, x_{2}\right]$ Hence, we have

$$
z(x)=z\left(x_{0}\right)=\|z\|_{C[0,1]}>1 \quad \text { for } x \in\left[x_{1}, x_{2}\right] .
$$

which contradicts the above property (iii).

Theorem 3.1 extends [6, Lemma 1(i)] or [26, Lemma 1.1(ii)] from $p=\sigma=2$ to $p \in(1, \infty)$ and $\sigma \in(1, p]$.

Acknowledgements. We would like to thank the anonymous referees for their careful reading on the manuscript and providing constructive comments.

\section{REFERENCES}

[1] R.P. Agarwal, H. Lü And D. O'Regan, Eigenvalues and the one-dimensional pLaplacian, J. Math. Anal. Appl. 266 (2002), 383-400.

[2] H. Amann, Fixed point equations and nonlinear eigenvalue problems in ordered Banach spaces, SIAM. Rev. 18 (1976), 620-709.

[3] D. Arcoya and D. Ruiz, The Ambrosetti-Prodi problem for the p-Laplacian, Comm. Partial Differential Equations 31 (2006), 849-865.

[4] E. Conway, R. Gardner and J. Smoller, it Stability and bifurcation of steady-state solutions for predator-prey equations, Adv. in Appl. Math. 3 (1982), 288-334.

[5] A. Ćwiszewski And M. MaCiejewski, Positive stationary solutions for p-Laplacian problems with nonpositive perturbation, J. Differential Equations 254 (2013), 1120-1136.

[6] E.N. DANCER, On positive solutions of some pairs of differential equations, Trans. Amer. Math. Soc. 284 (1984) (2), 729-743.

[7] G. DAI AND R. MA, Unilateral global bifurcation phenomena and nodal solutions for $p$ Laplacian, J. Differential Equations 252 (2012) (3), 2448-2468.

[8] M. del Pino, M. Elgueta and R. Manásevich, A homotopic deformation along $p$ of a Leray-Schauder degree result and existence for $\left(\left|u^{\prime}(x)\right|^{p-2} u^{\prime}(x)\right)^{\prime}+f(t, u)=0, u(0)=$ $u(T)=0, p>1$, J. Differential Equations 80 (1989), 1-13. 
[9] J. Dugundu and A. Granas, Fixed Point Theory, Springer-Verlag, 2004.

[10] A. Granas, The Leray-Schauder index and the fixed point theory for arbitrary ANRs, Bull. Soc. Math. France 100 (1972), 209-228.

[11] Z.M. GuO AND J.R.L. WeBB, Large and small solutions of a class of quasilinear elliptic eigenvalue problems, J. Differential Equations 180 (2002), 1-50.

[12] D.D. HAI, Existence and uniqueness of solutions for quasilinear elliptic systems, Proc. Amer. Math. Soc. 133 (2005), 223-228.

[13] D.D. Hai And H. WAng, Nontrivial solutions for p-Laplacian systems, J. Math. Anal. Appl. 330 (2007), 186-194.

[14] S. JUSTINO Multiple positive solutions of singular eigenvalue type problems involving the one-dimensional p-Laplacian, J. Math. Anal. Appl. 292 (2004), 401-414.

[15] R. KajikiYa, Y.H. LeE And I. Sim, One-dimensional p-Laplacian with a strong singular indefinite weight, I. Eigenvalue, J. Differential Equations 244 (2008), 1985-2019.

[16] _ Bifurcation of sign-changing solutions for one-dimensional p-Laplacian with a strong singular weight: p-superlinear at $\infty$, Nonlinear Anal. 74 (2011) (17), 5833-5843.

[17] _ Bifurcation of sign-changing solutions for one-dimensional p-Laplacian with a strong singular weight; p-sublinear at $\infty$, Nonlinear Anal. 71 (2009) (3-4), 1235-1249.

[18] Сн.-G. KIм, Existence, multiplicity and non-existence of positive solutions for two-point boundary-value problems with strong singularity, Proc. Roy. Soc. Edinburgh Sect. A $\mathbf{1 4 0}$ (2010), 1187-1196.

[19] D. Kinderlehrer and G. Stampacchia, Introduction to Variational Inequalities and Their Applications, Academic Press, New York, 1980.

[20] Q. Kong And M. Wang, Positive solutions of boundary value problems with p-Laplacian, Electron. J. Differential Equations 2010, No. 126, 16 pp.

[21] _ Nonlinear boundary value problems with p-Laplacian, Commun. Appl. Anal. 15 (2011) (1), 25-45.

[22] K.Q. LAN, Nonzero positive solutions of systems of elliptic boundary value problems, Proc. Amer. Math. Soc. 139 (2011), 4343-4349.

[23] K.Q. LAN AND Z. ZhANG, Nonzero positive weak solutions of systems of p-Laplace equations, J. Math. Anal. Appl. 394 (2012), 581-591.

[24] Y.H. LeE AND I. Sim, Existence of sign-changing solutions for one-dimensional pLaplacian problems with a singular indefinite weight, Topol. Methods Nonlinear Anal. 36 (2010) (1), 61-90.

[25] H.X. Li AND L.L. Zhang, On the sign-changing solutions for strong singular onedimensional $p$-Laplacian problems with p-superlinearity, Publ. Math. Debrecen 81 (2012) (3-4), 271-287.

[26] L.G. LI, Coexistence theorems of steady states for predator-prey interacting systems, Trans. Amer. Math. Soc. 305 (1988), 143-166.

[27] P. Lindqvist, On nonlinear Rayleigh quotients, Potential Anal. 2 (1993), 199-218.

[28] R.F. MANÁSEVICH AND F. ZANOlin, Time-mappings and multiplicity of solutions for the one-dimensional p-Laplacian, Nonlinear Anal. 21 (1993), 269-291.

[29] D. O'Regan and R. Precup, it Positive solutions of nonlinear systems with $p$-Laplacian on finite and semi-infinite intervals, Positivity 11 (2007) (3), 537-548.

[30] S. Oruganti, J. Shi and R. Shivaji, Diffusive equations with constant yield harvesting, I: steady states, Trans. Amer. Math. Soc. 354 (2002), 3601-3619.

[31] B.P. RYNNE, Eigenvalue criteria for existence of positive solutions of second-order, multipoint, p-Laplacian boundary value problems, Topol. Methods Nonlinear Anal. 36 (2010) (2), 311-326. 
[32] I. Sim, R. KAJIKIYA AND Y.H. LEe, On a criterion for discrete or continuous spectrum of p-Laplace eigenvalue problems with singular sign-changing weights, Nonlinear Anal. 72 (2010) (7-8), 3515-3534.

[33] J. WANG, The existence of positive solutions for the one-dimensional p-Laplacian, Proc. Amer. Math. Soc. 125 (1997) (8), 2275-2283.

[34] J.R.L. WebB, Positive solutions of nonlinear equations with explicit dependence on the independent variable, Differential and Difference Equations with Applications, Contributions from the International Conference on Differential \& Difference Equations and Applications, pp. 147-163 Series: Springer Proceedings in Mathematics \& Statistics, Vol. 47 (S. Pinelas, M. Chipot, Z. Dosla, eds.), 2013.

[35] _ Positive solutions of nonlinear equations via comparison with linear operators, Discrete Contin. Dyn. Syst. Ser. A 33 (2013) (11-12), 5507-5519.

[36] J.R.L. WEBB AND K.Q. LAN, Eigenvalue criteria for existence of multiple positive solutions of nonlinear boundary value problems of local and nonlocal type, Topol. Methods Nonlinear Anal. 27 (2006) (1), 91-116.

[37] X. YANG, Existence of positive solutions for quasi-linear differential equations, Comput. Math. Appl. 47 (2004), 1233-1239.

[38] Q.L. YAO, Existence, multiplicity and infinite solvability of positive solutions for onedimensional p-Laplacian, Acta Math. Sin. (Engl. Ser.) 21 (2005), 691-698.

[39] M.R. ZHANG, Nonuniform nonresonance at the first eigenvalue of the p-Laplacian, Nonlinear Anal. 29 (1997), 41-51.

[40] _ Nonuniform nonresonance of semilinear differential equations, J. Differential Equations 166 (2000), 33-50.

[41] Z. ZHANG AND S.J. LI, On sign-changing and multiple solutions of the p-Laplacian, J. Funct. Anal. 197 (2003), 47-468.

KUNQUAN LAN

Department of Mathematics

Ryerson University

Toronto, Ontario, M5B 2K3, CANADA

E-mail address: klan@ryerson.ca

XiAOJING YANG

Department of Mathematical Sciences

Tsinghua University

Bejing, 100084, P.R. CHINA

E-mail address: yangxj@mail.tsinghua.edu.cn

GuAnGChOng YANG

College of Mathematics

Chengdu University of Information Technology

Chengdu, Sichuan 610225, P.R. CHINA

E-mail address: gcyang@cuit.edu.cn 